\title{
Predictive role of plasma vascular endothelial growth factor for the effect of celecoxib in advanced non-small cell lung cancer treated with chemotherapy
}

Sverre Sörenson, Helena Fohlin, Andrea Lindgren, Magnus Lindskog, Bengt Bergman,

Christer Sederholm, Lars Ek, Kristina Lamberg and Birgitta Clinchy

\section{Linköping University Post Print}

N.B.: When citing this work, cite the original article.

Original Publication:

Sverre Sörenson, Helena Fohlin, Andrea Lindgren, Magnus Lindskog, Bengt Bergman, Christer Sederholm, Lars Ek, Kristina Lamberg and Birgitta Clinchy, Predictive role of plasma vascular endothelial growth factor for the effect of celecoxib in advanced non-small cell lung cancer treated with chemotherapy, 2013, European Journal of Cancer, (49), 1, 115120.

http://dx.doi.org/10.1016/j.ejca.2012.07.032

Copyright: Elsevier

http://www.elsevier.com/

Postprint available at: Linköping University Electronic Press http://urn.kb.se/resolve?urn=urn:nbn:se:liu:diva- 88362 


\title{
Predictive role of plasma vascular endothelial growth factor for the effect of celecoxib in advanced non-small cell lung cancer treated with chemotherapy
}

\author{
Sverre Sörenson ${ }^{\mathrm{a}, \mathrm{b}, *, \mathrm{n}}$, Helena Fohlin ${ }^{\mathrm{c}, \mathrm{d}, \mathrm{n}}$, Andrea Lindgren ${ }^{\mathrm{d}, \mathrm{e}, \mathrm{n}}$, Magnus Lindskog ${ }^{\mathrm{f}, \mathrm{g}, \mathrm{n}}$, \\ Bengt Bergman ${ }^{\mathrm{h}, \mathrm{i}, \mathrm{n}}$, Christer Sederholm ${ }^{\mathrm{b}, \mathrm{n}}$, Lars Ek ${ }^{\mathrm{j}, \mathrm{n}}$, Kristina Lamberg ${ }^{\mathrm{k}, \mathrm{n}}$, \\ Birgitta Clinchy ${ }^{1, \mathrm{~m}, \mathrm{n}}$
}

\footnotetext{
${ }^{a}$ Department of Medical and Health Sciences, Faculty of Health Sciences, Linköping University, Sweden

${ }^{\mathrm{b}}$ Department of Pulmonary Medicine UHL, County Council of Östergötland, Linköping, Sweden

${ }^{\mathrm{c}}$ Regional Cancer Centre, County Council of Östergötland, Linköping, Sweden

${ }^{\mathrm{d}}$ Department of Clinical and Experimental Medicine, Faculty of Health Sciences, Linköping University, Sweden

e Allergy Centre UHL, County Council of Östergötland, Linköping, Sweden

${ }^{\mathrm{f}}$ Department of Radiology, Oncology and Radiation Sciences, Uppsala University, Uppsala, Sweden

${ }^{\mathrm{g}}$ Department of Medical Sciences, Uppsala University, Uppsala, Sweden

${ }^{\mathrm{h}}$ Department of Respiratory Medicine, Sahlgrenska University Hospital, Gothenburg, Sweden

${ }^{\mathrm{i}}$ Institute of Medicine, University of Gothenburg, Sweden

${ }^{\mathrm{j}}$ Department of Respiratory Medicine and Allergology, Skåne University Hospital, Lund, Sweden

${ }^{\mathrm{k}}$ Department of Pulmonary Medicine, Uppsala University Hospital, Uppsala, Sweden

${ }^{1}$ Division of Clinical Immunology, Department of Clinical and Experimental Medicine, Faculty of Health Sciences, Linköping University, Sweden

${ }^{\mathrm{m}}$ Department of Clinical immunology and Transfusion Medicine, County Council of Östergötland, Linköping, Sweden
}

\section{KEYWORDS}

Non-small cell lung can-

cer

Celecoxib

Chemotherapy

Survival

Plasma VEGF

\begin{abstract}
Aim of the study: The primary purpose of this study is to investigate if pretreatment plasma levels of vascular endothelial growth factor (VEGF) are predictive of the effect of celecoxib on survival in advanced non-small cell lung cancer (NSCLC) treated with palliative chemotherapy. A secondary objective is to describe the course of plasma VEGF levels during and after treatment with cytotoxic chemotherapy combined with celecoxib or placebo.

Methods: In a previously published double-blind multicenter phase III trial, 316 patients with NSCLC stage IIIB or IV and World Health Organisation (WHO) performance status 0-2 were randomised to receive celecoxib $400 \mathrm{mg}$ b.i.d. or placebo in combination with two-drug platinum-based chemotherapy. Chemotherapy cycle length was three weeks and planned duration of chemotherapy was four cycles. Celecoxib was given for a maximum of one year but was stopped earlier in case of disease progression or prohibitive toxicity. In a subset of
\end{abstract}

\footnotetext{
* Corresponding author: Address: Bronsåldersgatan 15, Lgh 1202, SE-589 51 Linköping, Sweden. Tel.: +46 13161766

E-mail address: sverre.sorenson@liu.se (S. Sörenson).

${ }^{n}$ On behalf of the Swedish Lung Cancer Study Group.
} 
patients, plasma VEGF levels were examined at onset of treatment and at 6, 12 and 20 weeks. Results: VEGF levels at start of treatment were obtained in 107 patients at four study sites. The median value was $70 \mathrm{pg} / \mathrm{ml}$. Mean values declined during the first 12 weeks and then increased at 20 weeks. A subpopulation treatment effect pattern plot (STEPP) analysis showed an inverse relationship between initial plasma VEGF and the impact of celecoxib on survival with zero effect at $200 \mathrm{pg} / \mathrm{ml}$. The effect on survival by celecoxib in the whole subset of patients was positive (hazard ratio $(\mathrm{HR})=0.64$ [confidence interval (CI) 0.43-0.95], $p=0.028)$.

Conclusion: Low pretreatment plasma levels of VEGF appear to be predictive of a positive effect of celecoxib on survival.

(c) 2012 Elsevier Ltd. All rights reserved.

\section{Introduction}

The COX-2 inhibitor celecoxib has shown activity against non-small-cell lung cancer (NSCLC) in experimental systems. ${ }^{1,2}$ Two phase III studies investigating the effect on overall survival by adding celecoxib to chemotherapy in advanced NSCLC were, however, negative. $^{3,4}$ The negative overall results do not, however, exclude a clinical role of the compound in certain subpopulations. Since one of the mechanisms involved in the antineoplastic effect of COX-2 inhibitors appears to be inhibition of angiogenesis, ${ }^{5-7}$ biomarkers related to angiogenesis might be useful as predictors of a positive clinical effect on survival by celecoxib. Plasma levels of the angiogenic factor vascular endothelial growth factor (VEGF) have previously been shown to correlate to microvessel density in patients with NSCLC. ${ }^{8}$

There has been much debate as to which specimen, serum or plasma, is best suited for analysis of VEGF as a biomarker in cancer. ${ }^{9,10}$ Large amounts of VEGF can be released from platelets and leukocytes during clotting of serum and the amount released is dependent on clotting time and temperature. ${ }^{10,11}$ Hence, serum VEGF may represent blood platelet count rather than VEGF produced by other tissue. In this study we therefore chose to follow the levels of VEGF in plasma as this would be more likely to reflect tumour associated production of VEGF and be less sensitive to variations in sample handling than serum VEGF.

In the Swedish CYCLUS trial, comparing celecoxib with placebo in patients with advanced NSCLC receiving two-drug platinum-based chemotherapy, ${ }^{3}$ a subpopulation was examined with respect to plasma levels of VEGF before, during and after primary chemotherapy.

\section{Patients and methods}

Between May 2006 and May 2009, 316 patients with NSCLC stage IIIB-IV from 13 Swedish hospitals were included in a prospective randomised double-blind phase III trial comparing celecoxib at a dose of $400 \mathrm{mg}$ b.i.d. with placebo in combination with platinum-based chemotherapy. The details of the trial have been presented elsewhere. ${ }^{3}$ The drug regimens used by the participating hospitals were carboplatin + gemcitabine or carboplatin + vinorelbine. The cycle duration was three weeks and the planned number of cycles was four. Celecoxib or placebo was given from the first day of chemotherapy for a maximum of 1 year. Treatment with celecoxib/placebo was stopped earlier in case of disease progression, prohibitive toxicity related to the study drug or at the patient's wish.

At four hospitals that had accepted to do so, specimens for examination of plasma levels of VEGF were obtained before start of treatment, at 6,12 and 20 weeks. The VEGF analyses were approved by the regional ethics committee of the University of Linköping. All participating patients had given oral and written consent to the procedure.

\subsection{Laboratory methods}

\subsubsection{Blood samples}

Venous blood was collected in sterile vacutainer tubes using sodium EDTA as anticoagulant. The samples were kept on ice and centrifuged $\left(1500 \mathrm{~g}, 10 \mathrm{~min}, 4^{\circ} \mathrm{C}\right)$ within one hour of sampling. The plasma was aspirated into a centrifuge tube and centrifuged again $(3000 \mathrm{~g}, 10 \mathrm{~min}$, $4{ }^{\circ} \mathrm{C}$ ) to remove residual cells. The plasma was then transferred into polypropylene microcentrifuge tubes (Costar, Corning Incorporated Life Sciences, Lowell, MA) and stored at $-70{ }^{\circ} \mathrm{C}$ to $-80{ }^{\circ} \mathrm{C}$ until analysis.

\subsubsection{VEGF immunoassay}

Samples from sites outside Linköping were shipped over night on dry ice to the Laboratory of Clinical Immunology at the University Hospital in Linköping where all VEGF analyses were performed. The plasma was thawed at room temperature immediately before assay. The plasma levels of VEGF were analysed using a commercially available quantitative sandwich ELISA (Quantikine Human VEGF Immunoassay, R\&D Systems Europe Ltd., Abingdon, United Kingdom (UK)) according to the manufacturer's instructions. The assay measures both VEGF 165 and VEGF 121. The limit of sensitivity of the assay was $9.0 \mathrm{pg} / \mathrm{ml}$. Quantitation of VEGF in the samples was based on comparison to a linear standard curve ranging from 31.2 to $2000 \mathrm{pg} / \mathrm{ml}$ 
prepared with recombinant human VEGF supplied in the assay kit.

\subsection{Statistical methods}

Eighteen patients in the cohort had VEGF values below $31.2 \mathrm{pg} / \mathrm{ml}$, which cannot be quantified by the laboratory analysis kit used. In the statistical analysis where continuous values of VEGF were used, these values were set to 16.1 .

In order to understand the influence of VEGF on the treatment effect of celecoxib, a subpopulation treatment effect pattern plot (STEPP) analysis was performed. ${ }^{12}$ Rather than applying subgroup analysis based on arbitrary cut-off values of VEGF, the STEPP analysis is used to evaluate treatment effects with respect to VEGF as a continuous covariate. For the STEPP analysis the program stepp tail implemented in the statistical software Stata was used..$^{13}$ The parameter $g$ was set to 7 , generating 13 overlapping subgroups (six for decreasing VEGF values, one for all patients, and six for increasing VEGF values). The black solid curve in Fig. 4 shows the estimated log hazard ratio of celecoxib versus placebo in each of these subgroups. A curve value below zero indicates prolonged survival for the celecoxib group.

Overall survival was defined as the time from randomisation to death from any cause. Deaths until 31st March 2011 were included in the analysis. Failure probabilities were estimated according to the Kaplan-Meier method. Hazard ratios (HRs) and 95\% confidence intervals (CIs) were derived from Cox's proportional hazard regression.

Twenty-seven patients had VEGF values measured at all four time points, with specimens taken within an acceptable interval around $0,6,12$ and 20 weeks, respectively. To follow the trend of VEGF changes over time a longitudinal analysis using the program xtgraph implemented in Stata was performed. The graph shows the averages of VEGF with $95 \%$ confidence intervals measured at start of treatment, at 6 weeks, at 12 weeks and at 20 weeks.

All statistical analyses were performed using Stata/ SE 10.0.

\section{Results}

At least one specimen for determination of plasma VEGF was obtained in 107 out of 208 patients (51\%) who were randomised at the four hospitals. Clinical characteristics and the numbers of patients receiving celecoxib versus placebo are summarised in Table 1 . The proportion of patients with stage IV disease was slightly higher in the VEGF study group than in the CYCLUS study as a whole ( $82 \%$ versus $76 \%$ ) but the difference was not statistically significant. Other features were quite similar.

Mean values and confidence intervals of plasma VEGF levels that were obtained within acceptable intervals from $0,6,12$ and 20 weeks are displayed in Table 2. The median plasma level of VEGF before treatment was $70 \mathrm{pg} / \mathrm{ml}$. This value was used to divide patients into two groups with 'high' and 'low' initial plasma VEGF concentrations in subsequent analyses.

In 27 patients who underwent all four examinations, the course of mean values and confidence intervals is shown in Fig. 1. There was a gradual decline of mean values from elevated pretreatment levels up to week 12 , followed by an increase at week 20 , and the values at 6 and 12 weeks are significantly lower than before treatment. The course of VEGF concentrations was similar for responders and non-responders to treatment (Fig. 2) but baseline levels for VEGF were higher in the non-responder group.

In the whole subset of 107 patients, there was no significant survival difference between patients with initial VEGF concentrations $\geqslant$ or $<70 \mathrm{pg} / \mathrm{ml}$ (Fig. 3). Even progression-free survival was similar between the two groups (high versus low: $\mathrm{HR}=1.17$, CI $0.80-1.71$, $p=0.43$ ). The response rate was, however, significantly higher among patients with lower values $(43 \%$ versus $23 \%, p=0.028$ ).

Survival in patients with VEGF concentrations $<$ or $\geqslant 70 \mathrm{pg} / \mathrm{ml}$ was also compared in the two subgroups of patients treated with celecoxib or placebo, respectively. In patients treated with placebo $(n=55)$, the survival curves were virtually identical $(\mathrm{HR}=1.01$, CI $0.59-1.73, p=0.96$ ) for patients with low and high levels. In patients treated with celecoxib $(n=52)$, survival was slightly prolonged for patients with low values but the difference was not statistically significant (high versus low $\mathrm{HR}=1.36$, CI $0.77-2.41, p=0.29$ ).

Table 1

Clinical characteristics and treatment.

\begin{tabular}{ll}
\hline Characteristic & Number \\
\hline Age (median, range) & $65(37-84)$ \\
Male/female & $50 / 57$ \\
Stage IIIB/stage IV & $19 / 88$ \\
Performance status (WHO) 0/1/2 & $17 / 64 / 26$ \\
Smoker/ex-smoker/never smoker & $38 / 56 / 13$ \\
Squamous cell carcinoma/adenocarcinoma/other NSCLC & $16 / 65 / 26$ \\
Chemotherapy: carboplatin + vinorelbine/ & $66 / 37 / 4$ \\
$\quad$ carboplatin + gemcitabine/other & \\
Treatment with celecoxib/placebo & $52 / 55$ \\
\hline
\end{tabular}

Table 2

Median plasma levels and confidence intervals of vascular endothelial growth factor (VEGF) (pg/ml) before, during and after treatment with cytotoxic chemotherapy.

\begin{tabular}{lllll}
\hline $\begin{array}{l}\text { Time (weeks from } \\
\text { start of treatment) }\end{array}$ & Placebo & $n$ & Celecoxib & $n$ \\
\hline 0 & $102.8(75.1-130.6)$ & 55 & $107.3(74.2-140.3)$ & 52 \\
6 & $101.1(29.4-172.7)$ & 33 & $61.3(42.0-80.6)$ & 28 \\
12 & $32.5(21.1-43.9)$ & 23 & $55.3(29.6-80.9)$ & 19 \\
20 & $58.6(30.8-86.4)$ & 15 & $65.9(2.2-129.6)$ & 12 \\
\hline
\end{tabular}




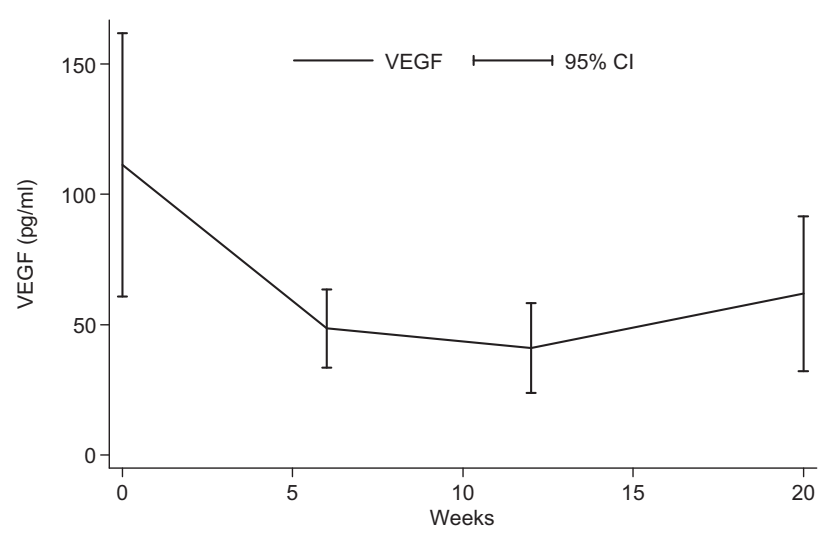

Fig. 1. Plasma vascular endothelial growth factor (VEGF) levels at 0 , 6, 12 and 20 weeks The diagram shows the subgroup of patients in whom specimens were available on all four occasions $(n=27)$. Mean values and $95 \%$ confidence intervals.

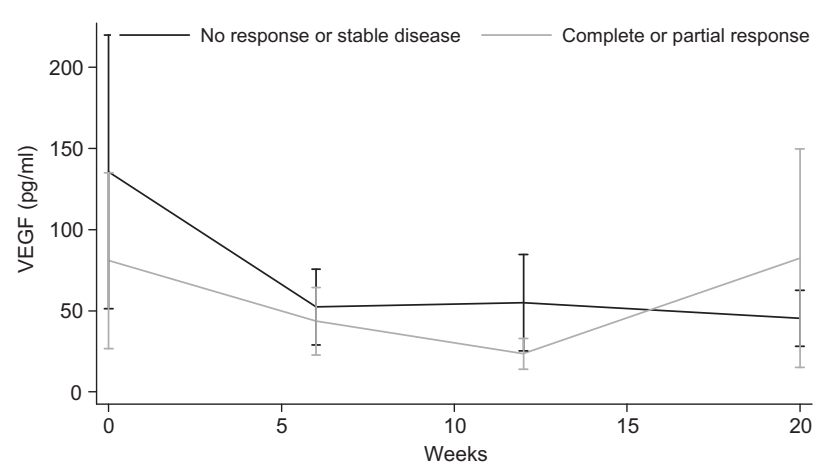

Fig. 2. Course of vascular endothelial growth factor (VEGF) levels with $95 \%$ confidence intervals according to response to treatment (complete and partial response versus stable disease and progression). Subset of 27 patients with all four measurements.

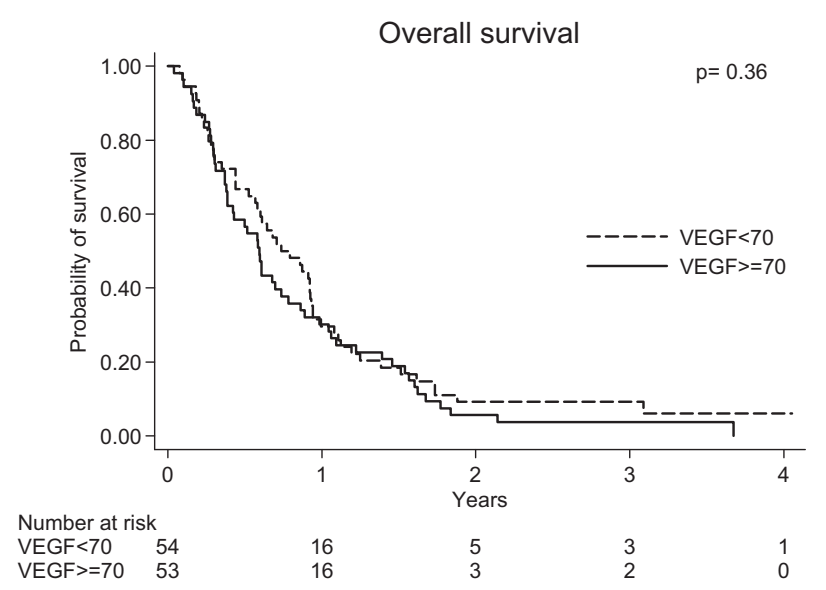

Fig. 3. Survival in patients with high $(\geqslant 70 \mathrm{pg} / \mathrm{ml})$ and low $(<70 \mathrm{pg} /$ $\mathrm{ml})$ pretreatment plasma vascular endothelial growth factor (VEGF) levels, respectively. Hazard ratio (HR) (high versus low) $=1.20(\mathrm{CI}$ $0.81-1.77), p=0.36$.

STEPP analysis showed an inverse relationship between initial plasma VEGF and the impact of celecoxib on survival with zero effect at $200 \mathrm{pg} / \mathrm{ml}$ (Fig. 4),

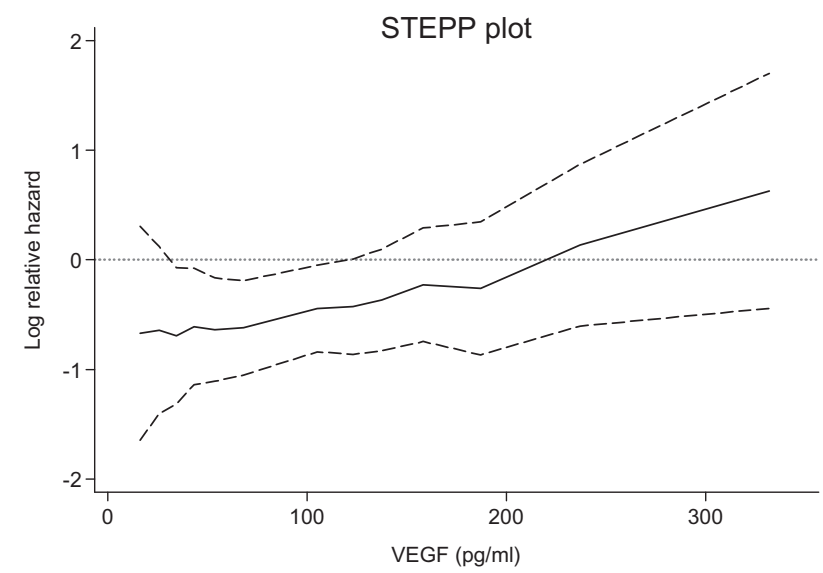

Fig. 4. Log hazard ratio for patients receiving celecoxib versus placebo as a function of initial plasma vascular endothelial growth factor (VEGF), with 95\% confidence intervals (subpopulation treatment effect pattern plot (STEPP) analysis). Low pretreatment VEGF concentrations are associated with a reduction of risk of death for patients treated with celecoxib.

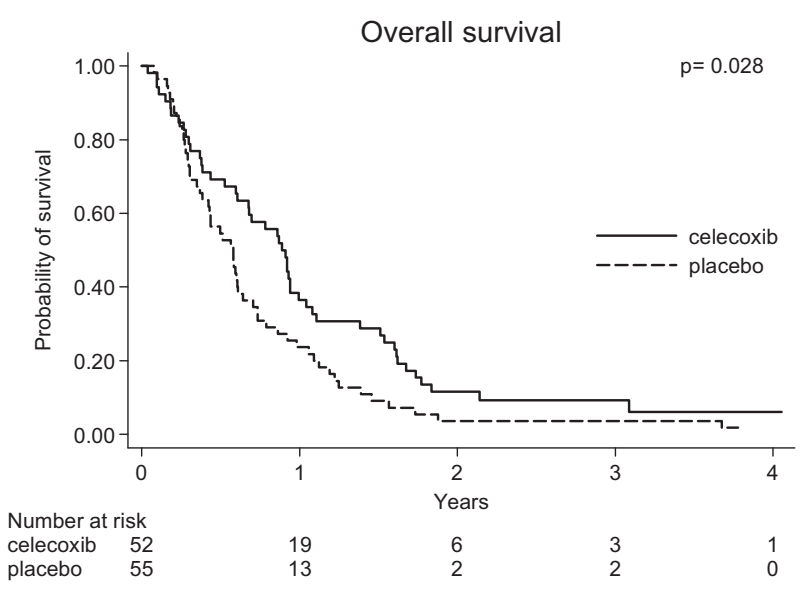

Fig. 5. Survival in patients treated with celecoxib versus placebo. Hazard ratio $(\mathrm{HR})=0.64(\mathrm{CI} 0.43-0.95), p=0.028$.

indicating a positive effect on survival by celecoxib as compared to placebo in patients with VEGF $<200 \mathrm{pg} /$ $\mathrm{ml}$ but not in patients with higher values. In the whole group, patients treated with celecoxib had significantly prolonged survival compared to patients treated with placebo (HR 0.64, CI $0.43-0.95, p=0.028$, Fig. 5). When survival with or without celecoxib is shown for patients with VEGF $\geqslant$ or $<100 \mathrm{pg} / \mathrm{ml}$ a significant survival gain with celecoxib versus placebo is confirmed for patients with lower levels while no such effect is seen in the other group (Fig. 6).

\section{Discussion}

The most important finding is an inverse relationship between initial plasma VEGF levels and the effect on 

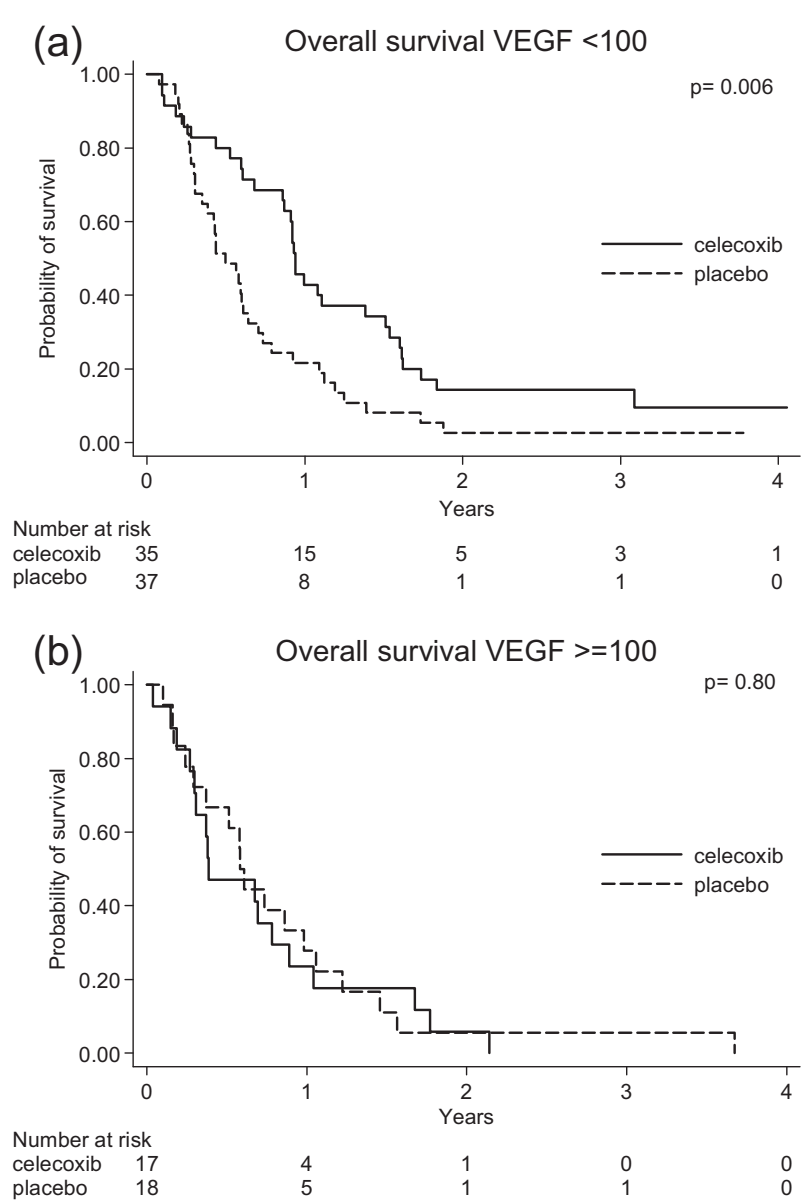

Fig. 6. (a and b) Overall survival in patients treated with celecoxib and placebo, respectively, with initial vascular endothelial growth factor (VEGF) levels $<100 \mathrm{pg} / \mathrm{ml}$ (hazard ratio (HR) $=0.50$ [CI 0.31-0.82] or $\geqslant 100 \mathrm{pg} / \mathrm{ml}(\mathrm{HR}=1.09$ [CI $0.55-2.15])$.

survival by the addition of celecoxib to chemotherapy. Low concentrations predict enhancement of survival while high concentrations do not. The observation is in accordance with data on vandetanib, an inhibitor of VEGFR-2, EGFR (epithelial growth factor receptor) and the RET proto-oncogene. Hanrahan and associates found that low pretreatment plasma VEGF concentrations were predictive of a positive effect on progression-free survival in patients with advanced NSCLC when vandetanib was compared with gefitinib or combined with docetaxel. ${ }^{14}$ In their study, high and low values were defined as levels $<$ or $\geqslant 115 \mathrm{pg} / \mathrm{ml}$, based on samples from healthy volunteers (data supplied by the manufacturer of the analysis kit). Median values for the patients in their study were, however, lower and in the same range as in our patients.

One possible reason for the predictive value of VEGF on the effect of celecoxib on survival in NSCLC could be that VEGF itself or other molecules expressed by VEGF-high-tumours, e.g. BCL-2 family proteins, might counteract antiproliferative or proapoptotic effects of celecoxib on the tumour endothelium or the lung cancer cells directly. ${ }^{15}$ Interestingly, preclinical findings indicate that VEGF may in fact rescue cancer cells from celecoxib-induced apoptosis ${ }^{16}$ and that anti-metastatic effects of coxibs may improve during simultaneous VEGF blockade. ${ }^{17} \mathrm{~A}$ reasonable hypothesis, therefore, is that VEGF might counteract antitumour effects of celecoxib and related drugs. Alternatively, lung tumours producing low or high levels of VEGF, respectively, might represent biologically different phenotypes that differ in their dependence on COX-2 or other targets of celecoxib.

We found an association between low pre-treatment plasma VEGF levels and response to chemotherapy as assessed by the individual investigator after completed first-line chemotherapy. This observation is in accordance with the findings of a previous smaller study. ${ }^{18}$ Another study found VEGFR-2 copy number gains in NSCLC resistant to platinum-based chemotherapy. ${ }^{19}$

We detected no association between pre-treatment VEGF levels and overall survival in the group as a whole (Fig. 3). Because low VEGF values were associated with a positive effect of celecoxib on survival, the analysis was repeated in the subgroups of patients treated with placebo and celecoxib, respectively. No statistically significant differences were observed but the numbers of patients were small. The absence of a prognostic role for plasma VEGF prior to treatment agrees with the results of a recent study where VEGF in plasma from 432 patients with advanced NSCLC treated with cisplatin and docetaxel was analysed and compared to 89 healthy age-matched controls. The mean plasma VEGF value in that study was $69.7 \mathrm{pg} / \mathrm{ml}$, which is lower than in our patients (Table 2). The authors demonstrated no survival differences albeit a significantly shorter time to progression in patients with adenocarcinoma and VEGF-A above the 75 th percentile. ${ }^{19}$ Hypothetically, VEGF may have a histology-dependent impact on prognosis in NSCLC. ${ }^{20}$ In the CYCLUS study, a large majority of patients had non-squamous histology.

Plasma VEGF levels showed a decline at 6 and 12 weeks, followed by an increase at 20 weeks (Fig. 1). These changes were, however, observed both in responders and non-responders which suggests a downregulation of VEGF production irrespective of tumour regression (Fig. 2).

An unexpected observation was a significantly positive effect of celecoxib on survival in the whole subpopulation analysed, in contrast to the result of the CYCLUS study as a whole. There is no obvious explanation for this finding. The clinical characteristics were similar for patients in the VEGF study and the whole CYCLUS population. A possible explanation could be that, for unknown reasons, the patients analysed with respect to VEGF had lower VEGF levels than average in the CYCLUS trial. 
In conclusion, plasma concentrations of VEGF appear to be a predictive factor for survival of patients with advanced NSCLC receiving combined treatment with celecoxib and standard palliative chemotherapy.

\section{Role of the funding source}

The study was supported by Östergötland County Council, Medical Research Council of South-East Sweden (FORSS). The funding source did not have any role in the study design, in the collection, analysis and interpretation of data, in the writing of the manuscript or in the decision to submit the manuscript for publication.

\section{Conflict of interest statement}

None declared.

\section{Acknowledgements}

We would like to thank the research nurses Gunilla Kaldenberg (Linköping), Izabella Sandberg (Linköping), Eva Quant (Gothenburg), Ing-Marie Nilsson (Lund), Helene Karlsson (Lund) and Ingemor Danielsson (Uppsala) for their help with taking, documenting and administrating the laboratory samples.

\section{References}

1. Liu X, Yue P, Zhou Z, Khuri FR, Sun SY. Death receptor regulation and celecoxib-induced apoptosis in human lung cancer cells. J Natl Cancer Inst 2004;96:1769-80.

2. Masferrer JL, Leahy KM, Koki AT, et al. Antiangiogenic and antitumor activities of cyclooxygenase- 2 inhibitors. Cancer Res 2000;60:1306-11.

3. Koch A, Bergman B, Holmberg E, et al. Effect of celecoxib on survival in patients with advanced non-small cell lung cancer: a double blind randomised clinical phase III trial (CYCLUS study) by the Swedish Lung Cancer Study Group. Eur J Cancer 2011;47:1546-55.

4. Groen HJ, Sietsma H, Vincent A, et al. Randomized, placebocontrolled phase III study of docetaxel plus carboplatin with celecoxib and cyclooxygenase- 2 expression as a biomarker for patients with advanced non-small-cell lung cancer: The NVALT-4 Study. J Clin Oncol 2011;29:4320-6.

5. Brown JR, DuBois RN. Cyclooxygenase as a target in lung cancer. Clin Cancer Res 2004;10:4266s-9s.
6. Leahy KM, Ornberg RL, Wang Y, et al. Cyclooxygenase-2 inhibition by celecoxib reduces proliferation and induces apoptosis in angiogenic endothelial cells in vivo. Cancer Res 2002;62:625-31.

7. Jones MK, Wang H, Peskar BM, et al. Inhibition of angiogenesis by nonsteroidal anti-inflammatory drugs: Insight into mechanisms and implications for cancer growth and ulcer healing. Nat Med 1999;5:1418-23.

8. Tamura M, Ohta Y, Kajita T, et al. Plasma VEGF concentration can predict the tumor angiogenic capacity in non-small cell lung cancer. Oncol Rep 2001;8:1097-102.

9. Jelkmann W. Pitfalls in the measurement of circulating vascular endothelial growth factor. Clin Chem 2001;47:617-23.

10. Hormbrey E, Gillespie P, Turner K, et al. A critical review of vascular endothelial growth factor (VEGF) analysis in peripheral blood: is the current literature meaningful? Clin Exp Metastasis 2002;19:651-63.

11. Webb NJ, Bottomley MJ, Watson CJ, Brenchley PE. Vascular endothelial growth factor (VEGF) is released from platelets during blood clotting: implications for measurement of circulating VEGF levels in clinical disease. Clin Sci (Lond) 1998;94:395-404.

12. Bonetti M, Gelber RD. A graphical method to assess treatmentcovariate interactions using the Cox model on subsets of the data. Stat Med 2000;19:2595-609.

13. Royston P, Sauerbrei W. A new approach to modelling interactions between treatment and continuous covariates in clinical trials by using fractional polynomials. Stat Med 2004;23:2509-25.

14. Hanrahan EO, Ryan AJ, Mann $\mathrm{H}$, et al. Baseline vascular endothelial growth factor concentration as a potential predictive marker of benefit from vandetanib in non-small cell lung cancer. Clin Cancer Res 2009;15:3600-9.

15. Karl E, Zhang Z, Dong Z, et al. Unidirectional crosstalk between $\mathrm{Bcl}-\mathrm{xL}$ and Bcl-2 enhances the angiogenic phenotype of endothelial cells. Cell Death Differ 2007;14:1657-66.

16. Catalano A, Graciotti L, Rinaldi L, et al. Preclinical evaluation of the nonsteroidal anti-inflammatory agent celecoxib on malignant mesothelioma chemoprevention. Int J Cancer 2004;109:322-8.

17. Fisher JC, Gander JW, Haley MJ, et al. Inhibition of cyclooxygenase 2 reduces tumor metastasis and inflammatory signaling during blockade of vascular endothelial growth factor. Vasc Cell 2011;3:22.

18. Kumar S, Guleria R, Singh V, Bharti AC, Mohan A, Das BC. Efficacy of plasma vascular endothelial growth factor in monitoring first-line chemotherapy in patients with advanced non-small cell lung cancer. BMC Cancer 2009;9:421.

19. Jantus-Lewintre E, Sanmartin E, Sirera R, et al. Combined VEGF-A and VEGFR-2 concentrations in plasma: diagnostic and prognostic implications in patients with advanced NSCLC. Lung Cancer 2011;74:326-31.

20. Pajares MJ, Agorreta J, Larrayoz M, et al. Expression of tumorderived vascular endothelial growth factor and its receptors is associated with outcome in early squamous cell carcinoma of the lung. J Clin Oncol 2012;30:1129-36. 